\title{
Die Medien aus der Sicht eines Nachrichtentechnikers
}

\author{
Bernd Eylert
}

\section{Zusammenfassung}

Der Autor hat zu diesem Thema am 8. Dezember 2010 am Institut für Medienwissenschaften der Philosophischen Fakultät an der Humboldt-Universität Berlin einen Vortrag gehalten. Es ist der Versuch eines Diskurses von Medien, Nachrichtentechnik und Philosophie, was unterscheidet sie, was verbindet sie, was haben sie gemein. Der Autor stellt besonders die Menschen in den Mittelpunkt, die diese Entwicklung maßgebend beeinflusst haben. Was sind Triebkraft und Motivation der Menschen, die mit ihrem Tun solch nachhaltige Veränderungen in der Gesellschaft bewirkt haben? Gibt es einen philosophischen Anspruch, dem sich Nachrichtentechniker verpflichtet fühlen? Der Beitrag versucht, auch darauf eine Antwort zu geben.

\section{Abstract}

The author has made a presentation to this item at the Institute for Media Sciences at the Faculty of Philosophy at Humboldt-University Berlin, $8^{\text {th }}$ December 2010. The paper is starting a discourse between media, telecommunications and philosophy. He points out what is different and what they do have in common. The author puts into his focus the people standing behind these developments. What has been the motivation for these people to push forward and change the society itself? Is there a philosophical guideline which is followed by these specialists? The author tries to give an answer.

\section{Einleitung}

Dieser Beitrag konzentriert sich auf solche Medien, die einen Nachrichtenkanal als Träger brauchen. Das ist zum ersten die drahtgebundene Kommunikation über Kabel oder Glasfaser direkt ins Haus, zum anderen die drahtlose Kommunikation, die vom Mobilfunk oder vom Rundfunk her bekannt ist, und endet beim Internet als der »All-inclusive«-Plattform. Damit wird sich klar von den Print- und Bildmedien abgegrenzt.

\section{Physikalische Grundlagen}

Die Existenz elektromagnetischer Wellen hat der schottische Physiker James Maxwell aufgrund mathematischer Berechnung vorhergesagt. Den Nachweis erbrachte Heinrich Hertz 1888. Bei seinem Versuch entdeckte er auch, dass ein solcher Schwingungsvorgang mit einem kurzen Stromstoß verbunden ist, der sich bei Hochspannung in einem Funken entlädt. Das Phänomen der Funkenbildung tritt an allen offenen Übergangsstellen zwischen zwei Polen auf. Die Bildung kleiner Funken ist letztlich dafür verantwortlich, dass elektromagnetische Wellen entstehen. Mittels dieser können wir über Antennen (Dipole) elektromagnetische Wellen abstrahlen, sodass wir Rundfunk, Fernsehen und Mobilfunk betreiben können.

Hertz, der Anglo-Italiener Gugliemo Marconi u. a. Physiker fanden heraus, dass man auf eine elektromagnetische Welle, einem Träger, elektromagnetische Wellen anderer Wellenlängen als Signale aufmodulieren kann. Damit war die Grundlage für die Übertragung von Nachrichten gelegt. Die ersten Sprachübertragungseinrichtungen, Telefon genannt, konnten entstehen. Maßgeblich umgesetzt in eine technische Anwendung hat es der schottisch-amerikanische Sprachtechniker und Stimmphysiologe Alexander Graham Bell. Entscheidend am Telefon sind zwei Dinge:

1. Die Umwandlung von Sprache in elektrische Signale und zurück.

2. Der Aufbau von Verbindungswegen, die eine Übermittlung von Nachrichten auch über große Strecken möglich machen.

Da Schritt 1 ein wichtiges Element zum physikalischen Verständnis der Telefonie ist, soll dies im Folgenden kurz 


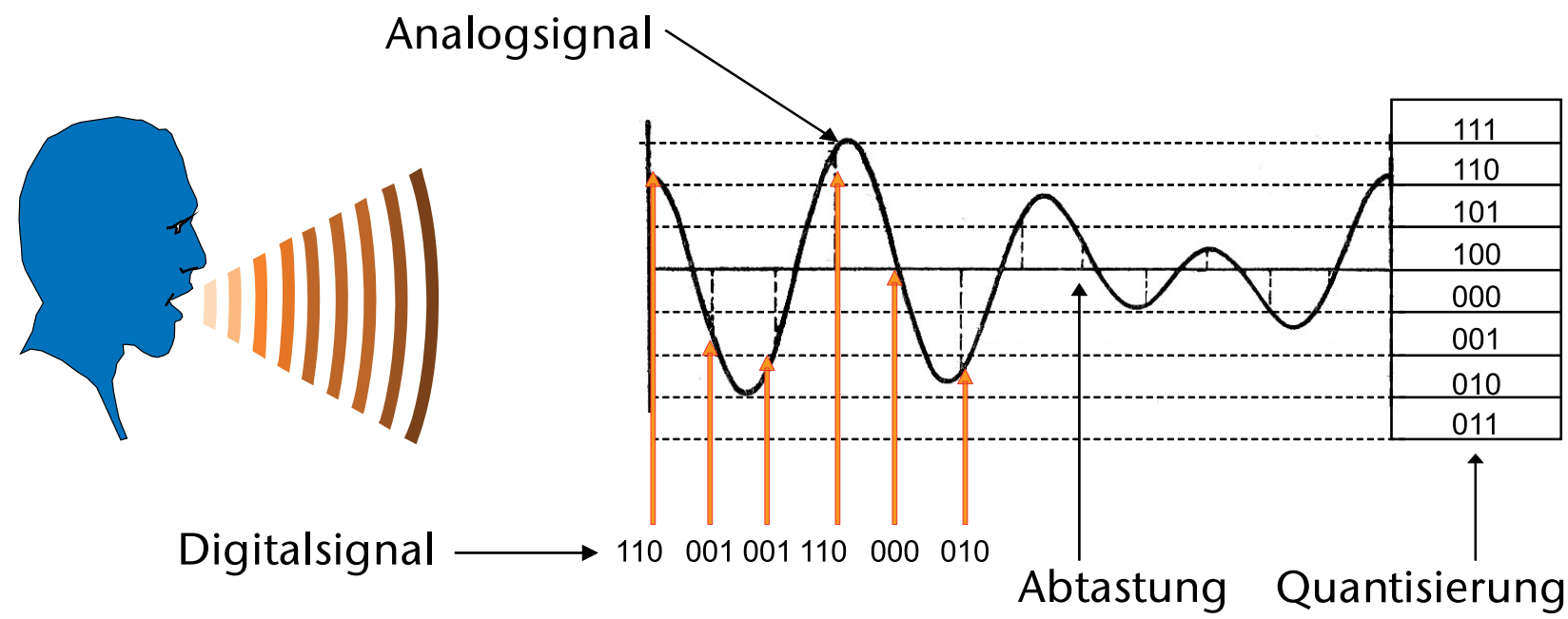

Abb. 1: Analog/Digital-Wandlung (Eylert 2010)

über die Analog/Digital-Wandlung erläutert werden. Dafür ist maßgeblich der amerikanische Mathematiker Claude Shannon verantwortlich. Er stieß damit die Tür für eine neue Entwicklung in der Nachrichtentechnik auf. Um dies ein wenig zu verdeutlichen, stellen wir uns vor, wir sprechen in ein Mikrofon (Eylert 2010: 176f.). Schallwellen werden 1:1 in elektrische Signale umgewandelt, was in Abb. 1 gezeigt wird.

Das wellenförmiges Signal, Analogsignal genannt, ist das Ergebnis der Umwandlung eines Schallsignals in ein elektrisches Signal, was im Mikrofon passiert. Jahrzehntelang hat man mit dieser Übertragungsart gut leben können. Claude Shannon kam auf die Idee, die Nachricht nicht mehr vollständig mit allen analogen Signalelementen zu übertragen, sondern nur noch ganz bestimmte Teile dieser Signale. Die Idee, die dahintersteckt, ist folgende: Die Sprache enthält eine Fülle von überflüssigen Lauten, die dem Sprachfluss, aber nicht dem Verständnis dienen. Man nennt das Redundanz. Das Gehirn wiederum ist in der Lage, fehlende Redundanz wieder dazu zu komponieren. Man kennt das von schlechten Telefonverbindungen, wo viele Worte verstümmelt werden, aber dennoch der Inhalt der Nachricht verstanden werden kann. Shannon benutzte für diesen Vorgang einen Quantisierer (rechte Seite im Bild) und ordnete dem Analogsignal einen binären Zahlenwert zu. D. h. man liest eine bestimmte Länge der Nachricht in einen Speicher ein, tastet anschließend das Analogsignal in gleichmäßigen Schritten ab und ordnet jedem einzelnen Abtastwert die entsprechende 1-0-Folge aus dem Quantisierer zu (s. Ziffernfolge unter der Kurve). Anschließend wird nur noch die 1-0-Folge, hier also »110 001001110000 010...«, übertragen. Auf der Empfängerseite geht der Prozess genau umgekehrt vor sich und lässt sich damit über einen Lautsprecher als Pendant zum Mikrofon in hörbare Sprache zurückverwandeln.

\section{Telefon und Funktelegraphie}

1877 baute Werner von Siemens das erste Telefonnetz in Berlin auf. Über Kupferdrähte wurden die Teilnehmer untereinander über eine Vermittlungsstelle verbunden. Die wichtigsten Personen der Gesellschaft und Behörden wurden angeschlossen. Es war zunächst ein sehr elitäres Netz, wurde aber zügig weiter ausgebaut und wer es sich leisten konnte, beantragte ein Telefon bei der Reichspost, denn diese war verantwortlich für den Telefondienst im Reich.

1899 kam es zur ersten Funkübertragung (drahtlose Telegraphie) über den Ärmelkanal durch Marconi, 1903 folgte die erste öffentliche transatlantische Funkverbindung durch Marconis Firma Marconi's Wireless Telegraph Company Ltd. Das System wurde von der britischen Marine übernommen.

Wollte man nicht beliebig viele Systeme weltweit einführen, so musste man sich auf Standards einigen. Dazu wurde schon im Jahre 1865 in Paris auf Anregung der französischen Regierung die Internationale Fernmeldeunion (Union Télégraphique Internationale) gegründet (International Telegraph Union 2011), wo bis heute die wesentlichen Standards in der Telekommunikation, einschließlich Rundfunk, Fernsehen, terrestrischer Mobil- und Satellitenfunk, vereinbart werden. Auf den Mobilfunk wird später noch einmal eingegangen. 


\section{Rundfunk (FV Sender Königs Wusterhausen e.V. 2011)}

Schon Anfang des letzten Jahrhunderts wurde in New York aus der Metropolitan oder seitens der Reichspost in Berlin aus der Oper unter den Linden versucht, über das Telefon Konzerte und Reden zu übertragen - mit mäßigem Erfolg. Denn die gebotene Qualität ersetzte noch keinen Konzertbesuch. Erste Rundfunkversuche hat es 1913 in New York gegeben. Die Versuche waren durchaus erfolgreich, wurden dann aber durch den beginnenden Ersten Weltkrieg unterbrochen.

In Deutschland führte ab April 1917 das Militär Erprobungen des Rundfunks auf dem Funkerberg in Königs Wusterhausen (kurz KW) unter Beteiligung des Physikers Alexander Meißner und des Ingenieurs Hans Bredow zur Übertragung von Sprache und Musik für Informations- und Unterhaltungssendungen durch. Die Versuche wurden abgebrochen, da die Vorgesetzten befohlen hatten, »diesen Unfug zu unterbinden « (Glaser/Werner 1990). Dass das nicht so unsinnig war, wie einige Heeresführer meinten, ist $u$. a. daran zu erkennen, dass der Erfolg der Russischen Revolution 1917 per »Rundspruch« an die kämpfenden Truppen verkündet wurde, was den Abschluss des später annullierten Separatfriedens von Brest-Litowsk und damit das Ende des Ersten Weltkrieges in Osteuropa nur beschleunigte.

Nach Beendigung des Ersten Weltkrieges übernahm die Deutsche Reichspost (DRP) die ehemalige Militärfunkstation und führte jetzt ihrerseits entsprechende Erprobungen unter Leitung des damals getadelten Hans Bredow durch. Am 22. Dezember 1920 wurde trotz Funkverbots der Alliierten erstmalig ein Weihnachtskonzert mit Instrumentalmusik, gespielt von Ingenieuren, Technikern und Angehörigen der DRP, ausgestrahlt, welches Zuschriften von privaten Zuhörern aus Luxemburg, Holland, England und den nordischen Staaten zur Folge hatte. Aus Deutschland kamen damals keine Reaktionen von Privathörern, da »Rundfunkhören« bei Strafe verboten war.

Zunächst wurde das neue Mittel für einen Wirtschaftsdienst angewendet, der 1921 ins Leben gerufen wurde. Telefunken und Lorenz entwickelten in Berlin die ersten Röhren. Röhren sind technische Bauelemente, die elektrische Signale verstärken und damit eine wirtschaftlich sinnvolle Übertragung ermöglichen können. Röhrensender wurden aufgebaut, Empfangsanlagen in Berlin-Zehlendorf errichtet und die Antennenanlage in KW um zwei weitere 100-Meter-Masten ergänzt. Es liefen also die Bemühungen der Post und das »Basteln « der Funk-Amateure nebeneinander her, wobei das Funkverbot der Alliierten die (offizielle) Freigabe des Empfangs für Amateure bis zum Jahre 1923 unmöglich machte. Erst am 29. Oktober 1923 konnte in Deutschland der "Unterhaltungsrundfunkdienst « als privatwirtschaftliche »Funkstunde $A G$ « starten, zwei Jahre nach dem Start in den USA und ein Jahr, nachdem die BBC in London erstmals auf Sendung gegangen war und eine beispiellose Medien-Karriere beginnt. Spiritus rector ist der Ingenieur Hans Bredow, jetzt Staatssekretär im Reichspostministerium.

Hier kommt aus Sicht der Nachrichtentechnik der erste entscheidende Einschnitt: In Deutschland übernehmen private Gesellschaften den Sendebetrieb. Nicht mehr Ingenieure und Physiker bestimmen und gestalten technisch und inhaltlich das Medium »Rundfunk «. Unternehmer beteiligen sich an den neuen Gesellschaften, die kommerziellen Interessen der Geldgeber stehen im Vordergrund. Publizisten gestalten die Programme und Ingenieure und Techniker sind für den Betrieb verantwortlich. Das soll an folgendem Beispiel verdeutlicht werden: Der für Köln bestimmte Sender musste zunächst in Münster arbeiten, weil die alliierte Rheinlandkommission das Empfangsverbot für das damals noch von Frankreich besetzte Rheinland aufrecht erhielt. Als publizistisches Mittel ersten Ranges erwies sich der Rundfunk in der Nacht zum 1. Februar 1926, als die alliierten Truppen das Rheinland verließen und es mit Hilfe der ersten Sendung aus Köln zu einer gewaltigen Volkskundgebung kam und man nun auch im Rheinland offiziell Rundfunk hören durfte.

Die Politik erkennt sofort die Bedeutung des Rundfunks für ihre Zwecke und überträgt am 1. März 1926 51 Prozent der Anteile der Reichs-Rundfunk-Gesellschaft (RRG) an die Reichspost. Diese ist gehalten, nach dem öffentlichen Grundsatz der sparsamen Haushaltsführung zu handeln, was nicht ohne Konflikte bleiben konnte. Bredow wird ab 1926 als Rundfunk-Kommissar der RRG als Vertrauensmann der Reichspost und der Rundfunkgesellschaften eingesetzt, übernimmt den Vorsitz im Verwaltungsrat und wird 1933 von den Nazis abgesetzt.

Ab 1933 untersteht der Rundfunk dem Propagandaministerium und bekommt damit eine völlig andere »Qualität«, was nach dem Zweiten Weltkrieg zu radikalen Änderungen führt. Die Post bleibt technischer Dienstleister. Die Entwicklung des Rundfunks in der 
Zeit von 1933 - $45 \mathrm{zu}$ beschreiben, ist ein eigenes, umfangreiches Thema, das den hiesigen Rahmen sprengen würde.

\section{Fernsehen}

(Deutsches Fernsehmuseum Wiesbaden 2010)

Nachdem der Rundfunk mit Sprach- und Musikübertragung erfolgreich eingeführt war, konnten sich die Nachrichtentechniker neuen Herausforderungen stellen: der Bildübertragung. Im Prinzip wiederholte sich die Entwicklung wie beim (Hör-)Rundfunk, nur mit einem wesentlichen Unterschied: In Deutschland wurde die Entwicklung des Fernsehens ganz wesentlich von den neuen Machthabern in Berlin gefördert und gesteuert. Sie wollten das Fernsehen aus propagandistischen Gründen zu den Olympischen Spielen 1936 in Berlin einsatzbereit haben.

Pionier des Fernsehens war zum einen der Ingenieur Paul Nipkow, der 1884 ein Verfahren erfand und patentierte, wie man (bewegte) Bilder zerlegen und anschließend übertragen kann. Ein weiterer Pionier des Fernsehens war Manfred von Ardenne. Er zeigte auf der 8. Großen Deutschen Funkausstellung 1931 in Berlin das System zur elektronischen Übertragung von Bildern auf Basis einer Kathodenstrahlröhre. Letztendlich ist es aber dem in Berlin lebenden Ungar Dénes von Mihály zu verdanken, dass das Fernsehen Marktreife erlangte. Er präsentierte auf der Funkausstellung in Berlin 1932 erstmals ein Gerät mit Bildröhre. Nach mehreren Versuchssendungen wurde am 22. März 1935 in Berlin das erste regelmäßige Fernsehprogramm Deutschlands gesendet. Gesendet wurde an drei Tagen in der Woche für jeweils zwei Stunden am Abend. Bereits 1936 wurde die erste Livesendung ausgestrahlt. Zu dieser Zeit gab es schätzungsweise 75 Heimempfänger, wie der Fernseher seinerzeit genannt wurde (Wegner 2008). Das Fernsehen war im Dritten Reich nicht so erfolgreich, wie man meinen könnte. Es heißt, den Machthabern wäre das Bild zu klein gewesen; sie wären nicht repräsentativ genug herübergekommen. Der Zweite Weltkrieg ließ das Fernsehen ab 1943 in Deutschland in einen sieben Jahre währenden Dornröschenschlaf fallen.

\section{Die elektronischen Massenmedien nach dem 2. Weltkrieg}

Nach dem 2. Weltkrieg übernehmen die Alliierten den gesamten Rundfunkbetrieb in Deutschland.

Aufgrund des Missbrauchs des Rundfunks als Propagandainstrument im Dritten Reich veranlassen die Alliierten 1948 die Trennung von Rundfunk und Staat in den westlichen Besatzungszonen. Gemäß Verordnung No. 118 der britischen Militärregierung von 1948 befiehlt sie die Einrichtung des Nordwestdeutschen Rundfunks (NWDR) in Hamburg, zuständig für die Britische Zone. Den technischen Betrieb übergibt sie an die Post. Sie legt auch fest, dass die Post die Rundfunkgebühren erhebt; 75 Prozent davon gehen an den NWDR für die Programmgestaltung und den redaktionellen Dienst und 25 Prozent an die Post für die technische Dienstleistung. Damit sind auch die Prioritäten zwischen der inhaltlichen Verantwortung und dem technischen Betrieb klar vorgegeben. Die anderen Besatzungsmächte handeln ähnlich, mit Ausnahme der Sowjets. In der sowjetischen Besatzungszone bleibt der Rundfunk Staatsaufgabe (Deutsches Fernsehmuseum Wiesbaden 2010).

Der Rundfunk wird durch das Grundgesetz (GG) Art. 5, 30, 70 und 73 sowie daraus folgendem Staatsvertrag Ländersache mit Ausnahme der in Art. 73 festgelegten Aufgaben des Bundes. Die Sender werden öffentlichrechtliche Einrichtungen, der Deutschen Bundespost obliegt der technische Betrieb und sie ist fiskalischer Dienstleister. Sie treibt nämlich bis zur Gründung der GEZ in den 1960er Jahren die Rundfunkgebühren selbst ein. In Teilen liegt die technische Dienstleistung bis heute in den Händen der Post-Nachfolgeorganisation, der Deutschen Telekom; viele Relaisstellen und Sender werden von ihr betrieben (Wegner 2008).

In der DDR bleibt der Rundfunk Staatsaufgabe, die Deutsche Post sichert den technischen Betrieb bis zur Wende 1990. Pikanterweise hat der Artikel 5 ein Pendant in der Verfassung der DDR, jedoch ohne den letzten Satz: »Eine Zensur findet nicht statt.« (Wegner 2008)

Das Fernsehen entstand in der alten Bundesrepublik erst wieder am 27.11.1950, ziemlich genau vor 60 Jahren, beim NWDR in Hamburg, wo mit dem Restteam der ehemaligen Reichspost-Fernseh-GmbH Testsendungen aufgenommen wurden. Weihnachten 1952 wurden die Fernsehsender Köln und Hannover in Betrieb genommen. Dank des Zusammenwirkens mit der Bundespost, die die Relaisstrecken ausbaute, und der 
Industrie konnten die Sender Berlin, Hamburg, Hannover, Köln und Langenberg in der Folgezeit das regelmäßige öffentliche Fernsehen ausstrahlen. Mit dem Vertragswerk »Deutsches Fernsehen« im März 1953 wurde für die 1950 gegründete Arbeitsgemeinschaft Deutscher Rundfunkanstalten (ARD) festgelegt, dass das Fernsehen eine Gemeinschaftsaufgabe ist. Die Beziehungen der Rundfunkanstalten zur Deutschen Bundespost hinsichtlich des Fernsehens wurden durch den Fernsehleitungsvertrag geregelt, den aber jede Anstalt Ende 1955separat mit der Post abschloss (Deutsches Fernsehmuseum Wiesbaden 2010).

In der DDR fand die erste Ausstrahlung am 21.12.1952 statt. Anlass war der 73. Geburtstag Stalins, der mit der ersten Nachrichtensendung der »Aktuellen Kamera «geehrt werden sollte. Offiziell startete der Deutsche Fernsehfunk (DFF) am 3.1.1956. Der gesamte technische Betrieb lag in den Händen der Deutschen Post. Mit Art. 36 des Einigungsvertrags von 1990 wurden die Auflösung des DFF und die Überführung ihrer Senderkette in die ARD zum 1.1.1992 festgelegt und umgesetzt. Die nördliche Kette ging im Norddeutschen Rundfunk (NDR) auf, im Süden entstand der Mitteldeutsche Rundfunk (MDR) und in Berlin-Brandenburg der Ostdeutsche Rundfunk Brandenburg (ORB), heute Rundfunk BerlinBrandenburg (RBB) (MDR 2011).

Sieht man einmal von der Entwicklung und Einführung des Farbfernsehens ab, wo es ja leidenschaftliche Auseinandersetzungen um den Standard zwischen den USA (NTSC), Frankreich (SECAM) und Deutschland (PAL) in den 1960er Jahren gab, war die große Zeit der Nachrichtentechniker in diesem Medium 1970 mehr oder weniger vorbei.

\section{UNO setzt über die Welthandels- organisation neue Schwerpunkte - WTO/GATS verändert die Märkte}

(Eylert 2005: 230ff.)

In den 1980er Jahren gab es nochmal ein Aufbäumen, als Satelliten- und Kabelfernsehen entwickelt und eingeführt wurden. Damit wurden neue Verbreitungskanäle eröffnet, die aufgrund der GATS-Vereinbarungen (General Agreement on Trade in Services) der World Trade Organisation (WTO) neue Maßstäbe durch grenzenlose Service-Dienstleistungen setzten und zur Einführung des Privaten Rundfunks führten. Neue Massenmedien entstanden. Mit verschlüsselten Programmen, ausgesendet von Satelliten, sollten mit massiver Werbung grenzüberschreitend neue Produkte vermarktet werden. Gleiches galt für das Geschäft auf den (koax-) Kabeln, das die DBP angestoßen, aber durch ihre eigene Privatisierung letztendlich nicht umsetzen konnte und als »nicht zum Kerngeschäft gehörend « verkaufen musste. Nach einer technischen Aufbauphase war auch das kein Feld für Nachrichtentechniker mehr. Sie verlagerten ihre Aktivitäten auf andere Gebiete, die neue Herausforderungen versprachen, z. B. den aufkommenden digitalen Mobilfunk.

\section{Entwicklung des Mobilfunks (Eylert 2005)}

Die Geschichte des Funks begann mit dem Seefunk. Bis zur Einführung des Mobilfunks liegt dazwischen aus nachrichtentechnischer Sicht eine beeindruckende Entwicklung, die aber aus der Sicht der Medien bis zum Jahr 1988 nicht so bedeutsam war. Auf zwei wichtige Meilensteine soll aber noch hingewiesen werden:

1. Die Entwicklung des Zugfunks und seine Einführung zur IFA 1925 in Berlin, der sich dadurch auszeichnete, dass er sich für den Kunden wie ein normales Telefonat darstellte, obwohl es über Langwelle im Gegensprechen, d. h. vollduplex, geführt wurde. Das war eine fantastische Leistung der Ingenieure der Deutschen Reichspost, die kein Pendant in der Welt hatte.

2. Die Entwicklung des Autotelefons, zunächst in den USA mit Einführung 1945 in St. Louis, dann in Deutschland sehr intensiv ab 1951 bei der Deutschen Bundespost, in den Regeldienst 1958 als A-Netz eingeführt. Ihm folgten noch die B- und C-Netze mit analoger Sprachübertragung, weshalb man sie auch als Mobilfunk der 1. Generation bezeichnet. Sieht man einmal vom skandinavischen Nordic Mobile Telephone (NMT) ab, waren alle Mobilfunknetze der 1. Generation nationale Funktelefonnetze.

\section{a) GSM}

Erstmalig hatten sich 198713 PTTs (nationale Post- und Fernmeldeorganisationen) und ein privater Netzbetreiber in einem Memorandum of Understanding (MoU) auf die Einführung eines gemeinsamen Mobilfunksystems nach dem GSM-Standard verständigt, eine Entwicklungsinitiative der Group Spéciale Mobile (GSM) und der CEPT (Conférence Européenne des Administrations des Postes et des Télécommunications). Keiner hatte 
auch nur ansatzweise die Idee, dass sich GSM als erstes digitales Mobilfunksystem weltweit durchsetzen würde. Schließlich war man ja aus Europa nicht gewöhnt, dass gemeinsame Ideen auch gemeinsam umgesetzt würden. Aber es gab ein sehr starkes politisches Committment, speziell aus Frankreich und Deutschland. Dieser deutsch-französischen Allianz, genannt D900 (digitaler Mobilfunk im 900MHz-Band), wollten andere EU-Staaten nicht tatenlos zusehen. GSM bekam später den Namen Global System for Mobile Communications und wurde als 2. Mobilfunkgeneration eingestuft.

\section{b) UMTS und der Weg zu LTE}

Die Europäische Union legte nach und das Forschungsund Entwicklungsprojekt RACE (Research in Advanced Communication Technologies in Europe), später Race I genannt, auf. Ziel dieses Forschungsprojektes war, Europa zum Weltmarktführer in Sachen Kommunikation zu machen. Für den Mobilfunk hieß das, aufbauend auf dem europäischen Standard GSM ein Universal Mobile Telecommunications System (UMTS) zu entwickeln. Ein neues Akronym war geboren. Nun galt es, dies mit Leben zu füllen. In diese neue Aufgabe eingebunden waren die PTTs, die Telekommunikationsindustrie und Forschungsinstitute (einschl. verschiedener Hochschulen). Umsetzen konnte man eine solche Aufgabe aber nur, wenn man die internationalen Standardisierungsund Normungsinstitute einband.

Was sollte die Systeme der 3. Mobilfunkgeneration von denen der 2. Mobilfunkgeneration unterscheiden? Bis einschließlich GSM ging es beim Funk rein um Sprachübertragung. An Datenübertragung über Funk hatte bis dato niemand gedacht. Solche Bandbreiten, wie wir sie für die Datenübertragung benötigen, galten im Funk bis dato als schwer realisierbar. Shannons
Kapazitätstheorem (s. Abb. 2), das im Wesentlichen eine Aussage zwischen dem Informationsgehalt einer Nachricht und der zur Verfügung stehenden Bandbreite zum Inhalt hatte, wurde zum Maßstab einer neuen Mobilfunkgeneration.

Der Mobilfunkdienst der 3. Generation (UMTS/3G) sollte eine Bildübertragung, Video-Conferencing, erlauben. Wenn eine Videokonferenz möglich ist, dann ist auch bidirektionales Fernsehen möglich! Aber mit welcher Bitrate? Ende der 1980er Jahre konnten Videokameras schon eine digitale Bildaufzeichnung mit $2 \mathrm{MBit} / \mathrm{s}$ (MPEG 2) realisieren. So entstand bei ITU-R WG 8F, der Arbeitsgruppe FPLMTS (Future Public Land Mobile Telecommunications System) der ITU, das Ziel, für 3G eine Datenübertragungsrate von mindestens 2 MBit/s (peak) zu schaffen.

Parallel dazu hatte sich in den 1990er Jahren das Internet als die Kommunikations- und Medienplattform entwickelt, die die Nutzung des klassischen Telefons dramatisch revolutionierte. Das Telefon wurde zu einem neuen Medium der weltweiten Kommunikation, weg von der reinen Sprachübertragung hin zu einem umfassenden Medienkanal. Waren am Anfang die Netzbetreiber auch noch Inhalteanbieter, so hat sich das im Laufe der letzten 15 Jahre auch durch die Vorgaben der EU dahingehend gewandelt, dass sich nun viele Diensteanbieter, sogenannte Service-Provider, auf den Netzen nicht immer zur Freude aller tummeln. Man denke da nur an die »Angebote« zur Kinderpornographie oder Sicherheitslücken im Netz, die gewiefte Geschäftemacher gnadenlos ausnutzen.

Abb. 2 verdeutlicht, wie sich die Peak-Datenraten in den letzten 20 Jahren vervielfacht haben (Scrase 2007). Während GSM eine sehr magere Datenrate vergleichbar der bekannten Faxübertragung im Festnetz bereitstell-
GSM/GPRS Evolution

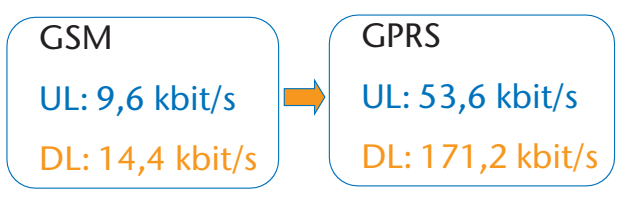

Shannon-Theorem:

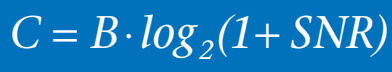

[3GPP Release'99

Release $6_{2004}$

Release $7_{2007}$

Release 8 $_{2009}$ ]

UMTS Evolution

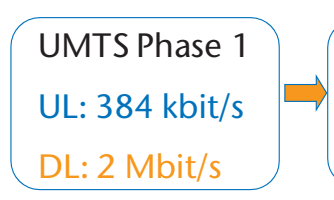

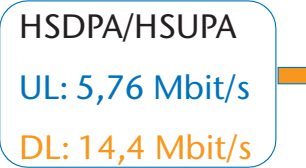

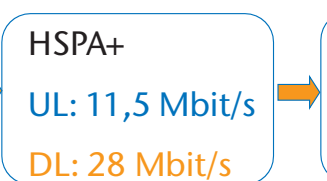

LTE (in $20 \mathrm{MHz}$ )

UL: $\leq 50 \mathrm{Mbit} / \mathrm{s}$

$\mathrm{DL}:<160 \mathrm{Mbit} / \mathrm{s}$ 


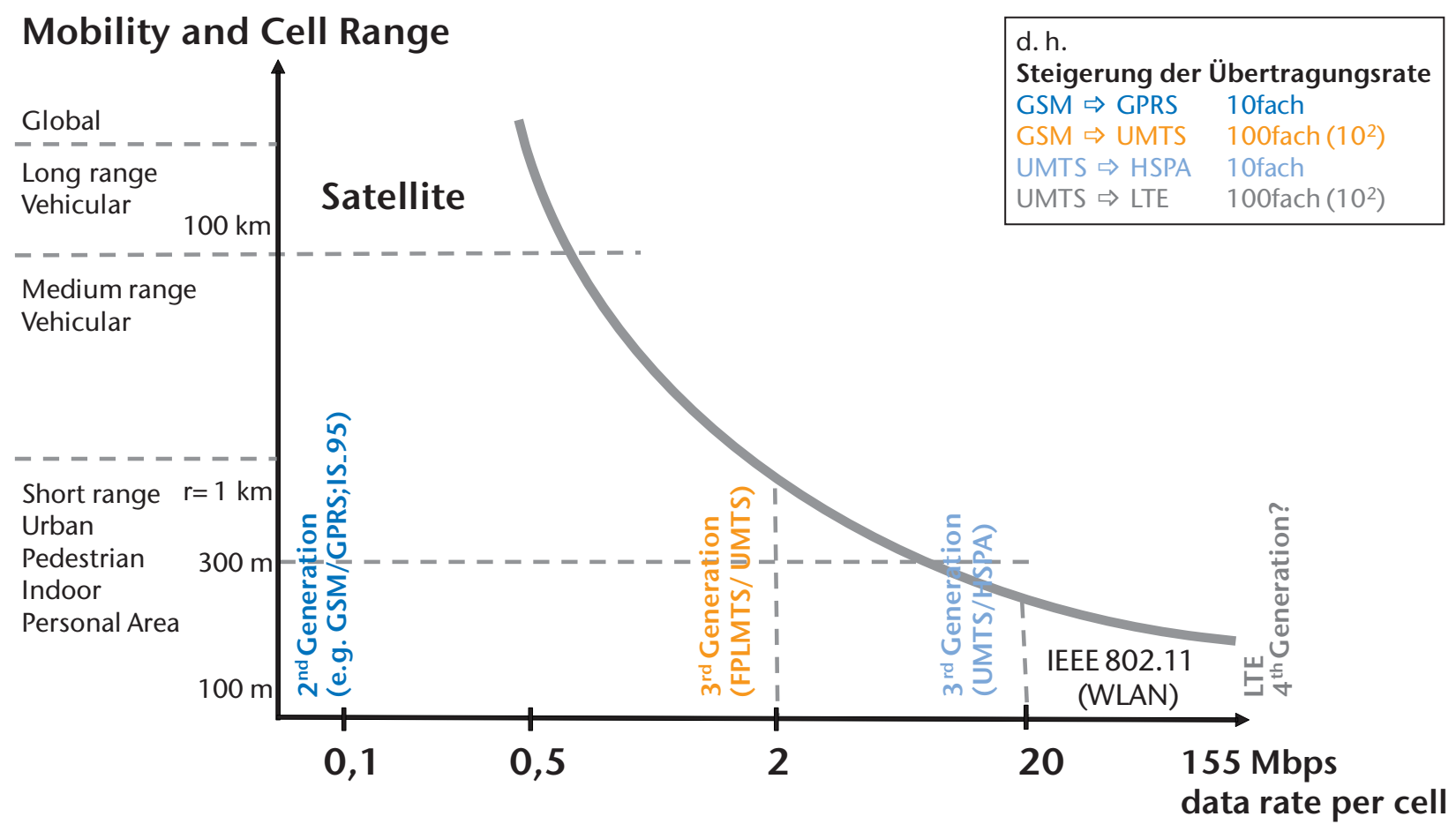

Abb. 3: Übertragungsraten in Abhängigkeit von der Reichweite

te, konnte GPRS (General Packet Radio Service) schon mit der Datenübertragungsrate bei ISDN konkurrieren. Das war zu Beginn der 1990er Jahre, als es noch kein Internet gab, noch okay. Mit dem Aufkommen des Internets und DSL im Festnetz musste das Mobilfunknetz nachziehen. Die Ziele von UMTS waren da durchaus adäquat. Mit der weiteren Leistungssteigerung und neuen Diensten aus dem Internet mit Video on Demand, PayTV etc., die das Festnetz dem Kunden anbot, kam eine weitere Herausforderung auf den Mobilfunk zu. Die Netzbetreiber sollten die WM 2006 in Deutschland als Chance begreifen, über UMTS Livestream-TV via Handy anzubieten. Es blieb bei Feldversuchen. 2008 zu den Olympischen Spielen in Peking wurde Livestream-TV via Handy zum Regeldienst, weil nun auch die fortgeschrittene Technologie HSPA (High Speed Package Access) eingeführt war, was als Bandbreite für kleine Bilddarstellungen ausreicht, heute noch zu sehen bei z. B. »Liga-Life «.

Technisch wird LTE (Long Term Evolution), von manchen auch schon als 4. Mobilfunkgeneration (4G) gesehen, diese und natürlich auch die Bedürfnisse der Bildübertragung ab 2011/12 voll erfüllen, sodass es für die Nachrichtentechniker an der Zeit ist, sich erneut neuen Herausforderungen zu stellen. Abb. 3 verdeutlicht die Übertragungsraten in Abhängigkeit von der Reichweite.

\section{Der philosophische Aspekt}

Es folgt der letzte Teil des Diskurses, der philosophische Aspekt nachrichtentechnischen Tuns und Handelns. Stellen wir uns daher zunächst einmal die Frage: Gibt es im Lebensplan von Nachrichtentechnikern allgemein, speziell bei Mathematikern, Physikern, Ingenieuren und Informatikern, einen philosophischen Grundkonsens, eine Grundidee, auf der sie ihr Lebenswerk aufbauen? Das ist bezogen auf die Neuzeit und die Moderne gar nicht so einfach herauszufinden. Stand bei Newtons Hauptwerk Philosophiae Naturalis Principia Mathematica noch Ad Maiorem Dei Gloriam als Leitspruch darüber, was seine philosophischen Wurzeln klar benannte, so findet man zwei Jahrhunderte später bei seinen Nachfolgern solche Bekenntnisse nicht mehr. Das ist sicherlich auch der Aufklärung und der Französischen Revolution mit der Trennung von Kirche und Staat geschuldet. Dennoch lohnt es sich, einen von ihnen herauszunehmen und ihn als pars pro toto zu betrachten: Heinrich Hertz (Hertz 1988).

Heinrich Hertz beginnt 1876 zunächst ein Ingenieur-Studium am Polytechnikum in Dresden mit dem Hauptaugenmerk auf Mathematik und Philosophie, wo er sich insbesondere mit Kants Kritik der Reinen Vernunft auseinandersetzt. Es ist nicht ungewöhnlich, dass sich viele Naturwissenschaftler in dieser Zeit, angeregt 
durch den Darwinismus, Kant, Hegel und Nitzsche zuwenden. Nüchterne Vernunft liegt ihnen näher als blumiges Pathos. Nach dem eingeschobenen einjährigen Militärdienst setzt er sein Studium 1877 am Polytechnikum in München fort, wechselt aber das Studienfach, um sich ganz den Naturwissenschaften zu widmen. Er studiert die Werke von Laplace, Lagrange und Newton. Besonders angetan haben es ihm aber die elliptischen Funktionen, ein außerordentlich spannendes und heute wieder hochaktuelles Spezialgebiet der Mathematik. Das bringt ihn dazu, 1878 an die Berliner Universität zu wechseln, um seine Studien bei den damaligen mathematischen Kapazitäten auf dem Gebiet - Weierstraß, Kummer und Kronecker - fortzusetzen und Physik bei Helmholtz und Kirchhoff zu studieren.

Helmholtz ermutigte ihn, sich 1879 an der jährlich ausgeschriebenen Preisarbeit der Philosophischen Fakultät mit einer Arbeit zum Wesen der Elektrizität zu beteiligen. Es ging letztendlich darum nachzuweisen, dass die Maxwellsche Theorie, Licht sei eine elektrische Welle, experimentell durch Messung der Ausbreitungsgeschwindigkeit der elektrischen Größen bestätigt wurde, was Hertz gelang und ihm den Preis einbrachte. In einer weiteren Preisarbeit, diesmal für die Akademie der Wissenschaften - die Aufgabenstellung kam wieder von Helmholtz - gelingt es Hertz, Maxwells Theorie vom Verschiebungsstrom nachzuweisen. Nun ist er wirklich in der Wissenschaft angekommen und schafft es, binnen drei Monaten seine Doktorarbeit, erste Darstellungen des Skineffekts, zu schreiben. Seine Verteidigung findet am 5. Februar 1880 statt. Dem Prüfungsausschuss gehören der Mathematiker Kummer, die Physiker Kirchhoff und Helmholtz und der Philosoph Zeller an. Müßig zu erwähnen, dass das Rigorosum eine wissenschaftliche Glanzvorstellung war. Man mag daran erkennen, welche Bedeutung der Philosophie im Gesamtkontext wissenschaftlicher Ausbildung noch vor über 100 Jahren beigemessen wurde.

Auch später hat die Philosophie Hertz nicht losgelassen. Neben all seinen experimentellen Arbeiten gilt es, die Ergebnisse in einen philosophischen Kontext einzubinden. Grundpfeiler damaliger Naturphilosophie war die Rückführung aller Vorgänge auf die klassische Mechanik. Dieser Grundgedanke lässt ihn bis zum Tode 1894 nicht los. In seinem letzten Buch, Die Prinzipien der Mechanik in neuem Zusammenhang dargestellt, von E. Lenard 1894 herausgegeben, versucht er, die Mechanik als Nahwirkungstheorie zu begründen und gibt immer wieder die philosophische Diskussion beeinflussende Einsichten. Andererseits muss er geradezu revolutionär auf seine Zeitgenossen gewirkt haben, wenn er auf die Frage, was die Maxwellsche Theorie sei, antwortete: »Die Maxwellsche Theorie ist das System der Maxwellschen Gleichungen.«Zu erkennen sind hier zwei wesentliche Ausprägungen der philosophischen Wahrheit. Einerseits der Logik folgend, sich erkenntnistheoretisch ausschließlich in der Übereinstimmung mit der objektiv widergespiegelten Realität konfrontiert zu sehen, und andererseits, metaphysisch sich in der Relation zwischen dem vom erkennenden Subjekt formulierten Aussage und dem Objekt selbst wiederzufinden.

Soviel zu Heinrich Hertz. Es ließen sich nun auch die anderen hier erwähnten Größen genauer ansehen. Dabei würde deutlich, dass bis in die 1960er Jahre das studium generale zur Grundausbildung eines jeden Studierenden gehörte. Es war verpflichtend, allgemeinbildende Vorlesungen zu den klassischen Gebieten Philosophie, Theologie, Naturwissenschaft, Medizin und Ökonomie zu hören. Die Studentenrevolten von 1968, da stehen besonders Paris, Berlin (West) und Frankfurt/M mit den Leitfiguren Jean-Paul Sartre, Jürgen Habermas und Ernst Jünger im Mittelpunkt, zielten auf ein neues Bildungsideal, den sozial engagierten, kritischen Bürger, der der Obrigkeit in allen Lebenslagen mit Widerspruch und Widerstand entgegentritt. Die Aufbaujahre in Westdeutschland waren zu Ende, die braune Vergangenheit noch nicht aufgearbeitet. Viele »reingewaschene « Altnazis saßen in vielen Bereichen des öffentlichen Lebens. Es wurde auf radikale Änderung gesonnen. Veränderungen machten an den Schulen und Hochschulen des Landes nicht halt.

Die gymnasiale Oberstufe wurde reformiert, Leistungskurse wurden geschaffen, die eher einer ersten »Fachausbildung « glichen. Das studium generale an den Hochschulen war nicht mehr verpflichtend, es verkümmerte oder entfiel ganz, ein neuer Zweig, die Soziologie, wurde zum Modefach der 1970er Jahre. In den Naturwissenschaften und technischen Studienrichtungen wurde verstärkt Wert auf eine gute Fachausbildung gelegt. Letzteres ist sicherlich wichtig, lässt aber einen wesentlichen Teil der menschlichen Bildung außen vor, die Auseinandersetzung mit den geistigen, ethischen und moralischen Grundlagen unserer Gesellschaft. Anstöße aus den Geisteswissenschaften gab es genug, sie verpufften aber schnell in den Curricula der MINT (Mathematik, Informatik, Naturwissenschaften, Technik)Wissenschaften. Einzig die Mathematiker als Mittler 
zwischen Geistes- und Naturwissenschaften und von der Logik herkommend, z. B. animiert durch den Tractatus Logico-Philosophicus von Ludwig Wittgenstein oder die Principia Mathematica von Alfred Whitehead und Bertrand Russel, fanden Zugang zu philosophischem Gedankengut.

Wo kommen wir her? Wo gehen wir hin? Ist unser Tun sachlich und moralisch gerechtfertigt? Wie steht es um die Ethik unseres Handelns? Diese Fragen werden für alle MINT-Wissenschaftler immer bedeutender. Wo waren sie, als in China oder im Iran der Zugang zum Internet eingeschränkt oder geblockt wurde? Oder im Arabischen Frühling, als 2011 z. B. in Ägypten oder in Syrien die Mobilfunknetze abgeschaltet wurden? Sicher, findige Köpfe haben Auswege über ausländische Server gefunden und Facebook hat einen unglaublichen Erfolg gefeiert. Hier muss auch an den Hochschulen im Studium der MINT mehr Bewusstsein geschaffen werden!

Der Autor hat diesen Faden aufgenommen und im Studiengang Telematik an der TH Wildau seine Studentinnen und Studenten im Seminar Telekommunikation und Gesellschaft mit der Philosophie konfrontiert. Dadurch werden sie motiviert, sich $u$. a. mit dem Sinn ihres Tun und Handelns und dem Sein als Ingenieur in dieser Gesellschaft auseinanderzusetzen. Für die meisten Studierenden ist dieser Ansatz zunächst sehr ungewöhnlich, geht es doch gar nicht um das bis dahin so erprobte Erlernen neuer Techniken und Verfahren. Am Ende des Semesters aber ist es nach ihrer Aussage - widergespiegelt in der den Studierenden allsemestrig abverlangten Evaluation - eine Bereicherung ihres Wissens und ihres Verantwortungsbewusstseins. Die dort gemachten Erfahrungen hätten sie nie für möglich gehalten und das neu erworbene Wissen möchten sie heute auch nicht mehr missen. Einen guten Einblick in das neue Denken ingenieurtechnischer Bildung spiegelt der Konferenzband You will shape the Digital Society with Your Knowledge - Make it Happen! an der TH Wildau wider (Eylert 2010).

\section{Fazit}

Die (neuen) Medien sind ohne die hervorragenden Leistungen der Nachrichtentechniker in den letzten rund 150 Jahren gar nicht denkbar. Sie haben die Grundlage für eine Entwicklung gelegt, die uns in die moderne Mediengesellschaft hineinkatapultiert hat. Vielfach wurden die neuen Medien von Menschen (z. B. Publizisten, Journalisten) übernommen und mit Inhalten gefüllt, die ihre Wurzeln in den Geisteswissenschaften haben. Für das erfolgreiche, zumindest aber gedeihliche Miteinander zum Wohle der Gesellschaft und ihrer Bildungsziele via neue Medien ist ein gutes Verständnis beider Bereiche notwendig. Junge Geistes- und Ingenieurwissenschaftler müssen also rechtzeitig an dieses Wissen und die Fähigkeiten, damit umzugehen, herangeführt werden. Zielführend handeln hier das Institut für Medientechnik an der Humboldt-Universität Berlin, das Institut für Medienwissenschaften an der Universität Erfurt und der Studiengang Telematik an der TH Wildau. Einerseits werden die Studierenden der Geisteswissenschaften behutsam an die Nachrichtentechnik herangeführt, sodass jede/r die Universität mit einem technischen Grundverständnis verlässt, andererseits werden Ingenieurstudierende mit der Philosophie vertraut gemacht und verlieren so die Scheu vor den Geisteswissenschaften. Damit wird beiden ermöglicht, über den eigenen Tellerrand zu schauen, ein höchst wünschenswerter Anspruch, der mehr Schule auch an anderen Hochschulen machen sollte.

\section{Literatur}

Deutsches Fernsehmuseum Wiesbaden (2010): Die Historie der "professionellen« Fernsehtechnik, http://www.fernsehmuseum.info/ vom-rundfunk-zum-fernsehen.html, Zugriff: 28.11.2010.

Eylert, B. (2005): The Mobile Multimedia Business.

Eylert, B. (2010): You will shape the Digital Society with Your Knowledge - Make it Happen!, Academic Farewell.

FV Sender Königs Wusterhausen e.V. (2011): Geschichte, http://www. funkerberg.de/geschichte, Zugriff: 03.11.2011.

Glaser, H., Werner, T. (1990): Die Post in ihrer Zeit.

Hertz, H. (1994): Festschrift anlässlich der Erforschung der elektromagnetischen Wellen vor 100 Jahren.

International Telegraph Union (2011): History, www.itu.int/en/about/ Pages/history, Zugriff: 03.11.2011.

MDR (2011): Fernsehen der DDR: Geschichte, www.mdr.de, Zugriff: 26.05.2011.

Scrase, A. (2007): Evolution of 3GPP's Wireless Standards, Presentation, TFH Wildau, 19.11.2007.

Wegner, R. (2008): Mobile TV - der lange Weg..., Vortrag, TFH Wildau, 10.11.2008. 


\section{Autor}

Prof. Dr.-Ing. Dipl.-Math. Bernd Eylert

Abteilungspräsident a.D.

Technische Hochschule Wildau [FH]

beylert@th-wildau.de 